\title{
User Cooperation in Wireless-Powered Backscatter Communication Networks
}

\author{
Bin Lyu, Dinh Thai Hoang, and Zhen Yang
}

\begin{abstract}
In this paper, we introduce new user-cooperation schemes for wireless devices in a wireless-powered backscatter communication network with the aim to improve communication and energy efficiency for the whole network. In particular, we consider two types of wireless devices which can support different communication modes, i.e., backscatter and harvestthen-transmit (HTT), and they can cooperate to deliver the information to the access point. To improve energy transmission efficiency for the devices, energy beamforming is deployed at the power beacon. We then formulate the weighted sum-rate (WSR) maximization problem by jointly optimizing time schedule, power allocation, and energy beamforming. Due to the non-convex issue of the optimization problem, we employ the variable substitutions and semidefinite relaxation (SDR) techniques to obtain the optimal solution. Simulation results show that the proposed cooperation framework can improve up to $33 \%$ communication efficiency compared with non-cooperation approach.
\end{abstract}

Index Terms-Energy harvesting, backscatter communication, user cooperation, energy beamforming.

\section{INTRODUCTION}

Wireless power transfer (WPT) has been considered to be a promising way to supply wireless devices with sustainable energy. In a wireless-powered communication network (WPCN), wireless devices can first harvest energy from a power beacon $(\mathrm{PB})$, and then transmit their information to the dedicated access point (AP) following the harvest-thentransmit (HTT) protocol [1]. In [2], [3], user cooperation was applied in WPCNs to enhance system performance by exploiting cooperative diversity. However, since both devices in [2], [3] are the HTT devices, the dedicated energy harvesting $(\mathrm{EH})$ phase is required, which may reduce the duration of the information transmission (IT) phase.

Recently, backscatter communication (BackCom) has been introduced as a novel communication method for IoT networks [4]. The BackCom device transmits information to the AP by modulating and reflecting the incident signals, which requires less circuit power consumption and makes its instantaneous harvested energy be sufficient to power its circuit operation [5]. Hence, the dedicated $\mathrm{EH}$ phase is not necessary, which avoids the limitation of the HTT protocol. However, one of the limitations of BackCom is that if the incident signal is unavailable, information backscattering (IB) is impossible. To fully exploit the advantages of both the HTT and BackCom, BackCom has been introduced in WPCNs [6], [7], where each device can choose to operate

B. Lyu and Z. Yang are with Nanjing University of Posts and Telecommunications, Nanjing 210003, China (email: \{blyu, yangz\}@ njupt.edu.cn).

D. T. Hoang is with University of Technology Sydney, Australia (email: hoang.dinh@uts.edu.au). in either the HTT or BackCom mode. However, IoT devices are energy-constrained and functionality-constrained devices, the backscatter and energy harvesting circuits and an adaptive switch required to support the HTT and BackCom modes may not be available in practice. Hence, the assumption that the devices can support both the two modes may not be practical. Furthermore, both user cooperation and energy beamforming are not considered in these works, hence the communication and energy efficiency can not be maximized.

In this paper, we introduce two user cooperation schemes for the WPCN with BackCom with the aim to optimize communication and energy efficiency for the network. In particular, we consider two wireless devices, denoted by HD and BD, supported to operate in two different modes, i.e., HTT and BackCom, respectively. We then consider two important scenarios, i.e., the $\mathrm{HD}(\mathrm{BD})$ is located nearer the $\mathrm{AP}$ and can be served as a relay node to assist the BD (HD) to transmit information due to the low channel quality of the $\mathrm{BD}$ (HD). For each scenario, we formulate the weighted sumrate (WSR) optimization problem by jointly optimizing the time schedule, power allocation, and energy beamforming. To deal with the non-convex issue of the optimization problem, we first employ the variable substitutions and design the optimal energy beamforming vector only for IB or information forwarding (IF). After that, the energy beamforming matrix is derived based on semidefinite relaxation (SDR) [11] for the joint IB and EH which satisfies the rank-one constraint. Simulation results then show that our proposed cooperation framework can achieve up to $33 \%$ communication efficiency than that of non-cooperation approach.

\section{SyStem MODEL AND NOTATIONS}

As illustrated in Fig. 1, we consider the WPCN with BackCom, including a PB, an AP, and two devices, denoted by $\mathrm{HD}$ and $\mathrm{BD}$, supported to operate in two different modes, i.e., HTT and BackCom, respectively. The PB with stable power supply has $N$ antennas, and the two devices are with single antenna. We consider two cases: (i) the HD is located nearer the AP than the BD, and it can operate as a relay node, and (ii) the $\mathrm{BD}$ is located nearer the AP than the HD, and it can work as a relay node. Note that the relay node also needs to deliver its own information to the AP. Moreover, we assume the relay node decodes the information transmitted by the other device more easily than the AP, which is useful for cooperative communication [2]. The channel vectors between the $\mathrm{PB}$ and the $\mathrm{BD} / \mathrm{HD} / \mathrm{AP}$ are denoted as $\boldsymbol{h}_{0,1}, \boldsymbol{h}_{0,2}$, and $\boldsymbol{h}_{0,3}$, respectively. The channel variables between the BD-HD, 


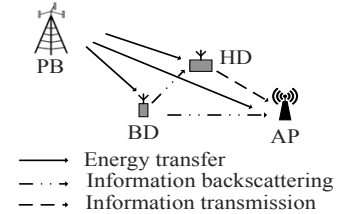

(a) Case $i$.

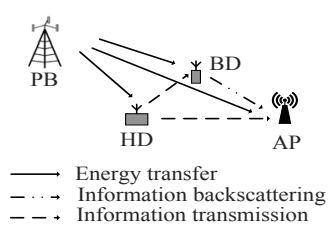

(b) Case ii.

Fig. 1: System model.

BD-AP, HD-AP, and HD-BD links are denoted as $h_{1,2}, h_{1,3}$, $h_{2,3}$, and $g_{1,2}$, respectively. Denote the received signal and signal-noise-ratio (SNR) at the $\mathrm{BD} / \mathrm{HD} / \mathrm{AP}$ during the $p+1$-th phase for Case $q$ as $y_{m, p, q}$ and $\gamma_{m, p, q}$, where $m=b d$, $h d, a p$, $p=0,1,2,3$, and $q=i, i i$. The achievable rates of the $\mathrm{BD} / \mathrm{HD}$ for Case $q$ is denoted by $R_{m, q}$. The system is considered within a normalized transmission time block, denoted by $T=1$.

\section{A. Case $i$ : the HD is located nearer the AP}

In this case, we divide the transmission block into four phases with duration denoted by $\tau_{i}(i=0, \cdots, 3)$, where $\sum_{i=0}^{3} \tau_{i} \leq 1$. During $\tau_{0}$, the BD backscatters information to the AP, while the HD harvests energy. Denote the transmitted signal of the PB during $\tau_{0}$ as $\boldsymbol{w}_{0, i}(\tau)$ which is expressed by $\boldsymbol{w}_{0, i}(\tau)=\sqrt{P} \hat{\boldsymbol{w}}_{0, i} s(\tau)$, where $P$ is the transmit power of the $\mathrm{PB}, s(\tau)$ is a known sequence with unit power, and $\hat{\boldsymbol{w}}_{0, i}$ is the energy beamforming vector during $\tau_{0}$ and satisfies $\left\|\hat{\boldsymbol{w}}_{0, i}\right\|^{2} \leq 1$. The received signal at the BD during $\tau_{0}$, denoted by $u_{0, i}(\tau)$, is expressed as $u_{0, i}(\tau)=\boldsymbol{h}_{0,1}^{H} \boldsymbol{w}_{0, i}(\tau)+n_{a n}(\tau)$, where $n_{a n}(\tau)$ is the antenna noise. Denote the own signal of the $\mathrm{BD}$ for Case $i$ as $c_{i}(\tau)$, which is modulated on $u_{0, i}(\tau)$ by controlling the reflection coefficient $\alpha_{0, i}$, where $\mathbb{E}\left[\left|c_{i}(\tau)\right|^{2}\right]=1, \alpha_{0, i}$ is a complex coefficient and $\left|\alpha_{0, i}\right|^{2} \leq 1$. The received signal at the AP during $\tau_{0}$ is then given by $y_{a p, 0, i}(\tau)=\sqrt{P} \alpha_{0, i} h_{1,3} \boldsymbol{h}_{0,1}^{H} \hat{\boldsymbol{w}}_{0, i} s(\tau) c_{i}(\tau)+h_{1,3} \alpha_{0, i} c_{i}(\tau) n_{a n}(\tau)+$ $\sqrt{P} \boldsymbol{h}_{0,3}^{H} \hat{\boldsymbol{w}}_{0, i} s(\tau)+n_{a p}(\tau)$, where $h_{1,3} \alpha_{0, i} c_{i}(\tau) n_{a n}(\tau)$ is the noise backscattered to the AP, $\sqrt{P} h_{0,3}^{H} \hat{\boldsymbol{w}}_{0, i} s(\tau)$ is the interference signal from the $\mathrm{PB}, n_{a p}(\tau)$ represents the additive white Gaussian noise (AWGN) with zero mean and variance $\sigma_{a p}^{2}$. The backscattered noise power is much smaller than that of $n_{a p}(\tau)$ due to channel attenuation and is typically negligible. Moreover, since the AP can also receive $\hat{w}_{0, i} s(\tau)$, the perfect self-interference cancellation (SIC) technique ${ }^{1}$ is employed to subtract the interference signal from $y_{a p, 0, i}(\tau)$ [8]. The SNR at the AP during $\tau_{0}$ is thus expressed as $\gamma_{a p, 0, i}=$ $P\left|\alpha_{0, i}\right|^{2}\left|h_{1,3}\right|^{2}\left|\boldsymbol{h}_{0,1}^{H} \hat{\boldsymbol{w}}_{0, i}\right|^{2} / \sigma_{a p}^{2}$. Similarly, the received powers from the backscattered signal and noise at the HD are much smaller than those of the PB and are negligible. Hence, the harvested energy at the HD is given by $E_{h, i}=\eta P\left|\boldsymbol{h}_{0,2}^{H} \hat{\boldsymbol{w}}_{0, i}\right|^{2} \tau_{0}$, where $\eta$ is the energy harvesting efficiency.

During $\tau_{0}$, the direct $\mathrm{IB}$ rate from the $\mathrm{BD}$ to the $\mathrm{AP}$ may be limited due to the energy beamforming tradeoff between IB and EH and the far distance between the BD and the AP. Hence, during the second and third phases, the

\footnotetext{
${ }^{1}$ Generally, the interference can not be canceled completely. However, even if there exists the residual interference after cancellation, the structures and conclusions of the aftermentioned results will not be changed.
}

HD operates as a relay node to transmit IF of the BD, where the HD first receives the backscattered signal and then forwards it to the AP via decode-and-forward (DF) operation following [2]. Since the transmitted signal at the PB during $\tau_{1}$ only focuses on IB of the $\mathrm{BD}$, we let the normalized energy beamforming vector be $\hat{\boldsymbol{w}}_{1, i}$. The transmitted signal is thus expressed as $\boldsymbol{w}_{1, i}(\tau)=\sqrt{P} \hat{\boldsymbol{w}}_{1, i} s(\tau)$. The backscattered signal is received by both the HD and the AP, and SIC is operated. The SNRs at the HD and the AP during $\tau_{1}$ are respectively given by $\gamma_{h d, 1, i}=P\left|\alpha_{0, i}\right|^{2}\left|h_{1,2}\right|^{2}\left|\boldsymbol{h}_{0,1}^{H} \hat{\boldsymbol{w}}_{1, i}\right|^{2} / \sigma_{h d}^{2}$ and $\gamma_{a p, 1, i}=P\left|\alpha_{0, i}\right|^{2}\left|h_{1,3}\right|^{2}\left|\boldsymbol{h}_{0,1}^{H} \hat{\boldsymbol{w}}_{1, i}\right|^{2} / \sigma_{a p}^{2}$, where $\sigma_{h d}^{2}$ is the noise power at the HD. During the third phase, the HD decodes the received signal from the $\mathrm{BD}$ [9] and forwards it to the AP. The forwarded signal received by the AP during $\tau_{2}$ is expressed as $y_{a p, 2, i}(\tau)=\sqrt{P_{1, i}} h_{2,3} c_{i}(\tau)+n_{a p}(\tau)$, where $P_{1, i}$ is the transmit power of the HD for IF, and the SNR is expressed as $\gamma_{a p, 2, i}=P_{1, i}\left|h_{2,3}\right|^{2} / \sigma_{a p}^{2}$. During the fourth phase, the HD transmits its own information to the AP. Similarly, the SNR at the AP during $\tau_{3}$ is expressed as $\gamma_{a p, 3, i}=P_{2, i}\left|h_{2,3}\right|^{2} / \sigma_{a p}^{2}$, where $P_{2, i}$ denotes the HD's transmit power for its own IT.

Based on the above analysis, the achievable rates of the BD and the HD are expressed as $R_{b d, i}=\tau_{0} \log _{2}\left(1+\xi \gamma_{a p, 0, i}\right)+$ $\min \left\{\tau_{1} \log _{2}\left(1+\xi \gamma_{a p, 1, i}\right)+\tau_{2} \log _{2}\left(1+\xi \gamma_{a p, 2, i}\right), \tau_{1} \log _{2}(1+\right.$ $\left.\left.\xi \gamma_{h d, 1, i}\right)\right\}$ [10] and $R_{h d, i}=\tau_{3} \log _{2}\left(1+\xi \gamma_{a p, 3, i}\right)$, respectively, where $\xi$ is the performance gap due to the practical modulation and coding scheme [1], [7]. Note that since there exists the backscattered noise at the AP, the above expressions are the approximation of the real transmission rate of the BD [5].

\section{B. Case ii: the BD is located nearer the AP}

In the second case, we divide the transmission block into three phases with duration denoted by $t_{i}(i=0,1,2)$, where $\sum_{i=0}^{2} t_{i} \leq 1$. During the first phase, the HD harvests energy and the BD backscatters information. Denote the normalized energy beamforming vector, the own signal of the $\mathrm{BD}$, and the reflection coefficient during $t_{0}$ for Case $i i$ as $\hat{\boldsymbol{w}}_{0, i i}$, $c_{i i}(t)$, and $\alpha_{0, i i}$, respectively, where $\mathbb{E}\left[\left|c_{i i}(t)\right|^{2}\right]=1$ and $\left|\alpha_{0, i i}\right|^{2} \leq 1$. The harvested energy at the HD is given by $E_{h, i i}=\eta P\left|\boldsymbol{h}_{0,2}^{H} \hat{\boldsymbol{w}}_{0, i i}\right|^{2} t_{0}$. Similarly, the backscattered noise is not considered and the SIC is adopted in this case. The SNR at the AP during $t_{0}$ is thus given by $\gamma_{a p, 0, i i}=$ $P\left|\alpha_{0, i i}\right|^{2}\left|h_{1,3}\right|^{2}\left|\boldsymbol{h}_{0,1}^{H} \hat{\boldsymbol{w}}_{0, i i}\right|^{2} / \sigma_{a p}^{2}$. During the last two phases, the IT of the HD is transmitted by the BD. During the second phase, the HD transmits its information to both the AP and the BD based on the harvested energy, and the PB keeps idle. The SNRs at the AP and the BD are thus given by $\gamma_{a p, 1, i i}=$ $P_{1, i i}\left|h_{2,3}\right|^{2} / \sigma_{a p}^{2}$ and $\gamma_{b d, 1, i i}=P_{1, i i}\left|g_{1,2}\right|^{2} / \sigma_{b d}^{2}$, where $P_{1, i i}$ is the transmit power of the HD and satisfies $P_{1, i i} t_{1} \leq E_{h, i i}$, and $\sigma_{b d}^{2}$ is the noise power at the BD. During the third phase, the $\mathrm{PB}$ is activated, and the $\mathrm{BD}$ can forward the information from the HD to the AP via DF. The subsequent SNR after SIC is given by $\gamma_{a p, 2, i i}=P\left|\alpha_{2, i i}\right|^{2}\left|h_{1,3}\right|^{2}\left|\boldsymbol{h}_{0,1}^{H} \hat{\boldsymbol{w}}_{2, i i}\right|^{2} / \sigma_{a p}^{2}$, where $\hat{\boldsymbol{w}}_{2, i i}$ is the normalized energy beamforming vector during $t_{2}$, $\alpha_{2, i i}$ is the reflection coefficient during $t_{2}$ and $\left|\alpha_{2, i i}\right|^{2} \leq 1$.

Then, the achievable rates of the HD and the BD for Case ii are given by $R_{h d, i i}=\min \left\{t_{1} \log _{2}\left(1+\xi \gamma_{a p, 1, i i}\right)+t_{2} \log _{2}(1+\right.$ $\left.\left.\xi \gamma_{a p, 2, i i}\right), t_{1} \log _{2}\left(1+\xi \gamma_{b d, 1, i i}\right)\right\}$, and $R_{b d, i i}=t_{0} \log _{2}(1+$ $\left.\xi \gamma_{a p, 0, i i}\right)$. 


\section{Weighted SUM-RATE MAXimization}

\section{A. Case $i$}

We first set the time and energy constraints for network as follows: $\mathrm{C} 1: \sum_{i=0}^{3} \tau_{i} \leq 1, \mathrm{C} 2: \tau_{i} \geq 0, \forall i, \mathrm{C} 3: P_{1, i} \tau_{2}+$ $P_{2, i} \tau_{3} \leq E_{h, i}, \mathrm{C} 4:\left\|\hat{\boldsymbol{w}}_{0, i}\right\|^{2} \leq 1$, and C5: $\left\|\hat{\boldsymbol{w}}_{1, i}\right\|^{2} \leq 1$. Then, the optimization problem can be formulated by

$$
\begin{aligned}
\max _{\hat{\boldsymbol{w}}_{0, i}, \hat{\boldsymbol{w}}_{1, i}, \boldsymbol{P}_{i}, \boldsymbol{\tau}} & \omega_{1} R_{b d, i}+\omega_{2} R_{h d, i} \\
\text { s.t. } & \mathrm{C} 1, \mathrm{C} 2, \mathrm{C} 3, \mathrm{C} 4, \mathrm{C} 5,
\end{aligned}
$$

where $\boldsymbol{\tau}=\left[\tau_{0}, \tau_{1}, \tau_{2}, \tau_{3}\right], \boldsymbol{P}_{i}=\left[P_{1, i}, P_{2, i}\right], \omega_{1}$ and $\omega_{2}$ denote the nonnegative rate weights for the $\mathrm{BD}$ and the HD, respectively. Problem P1 is not a convex optimization problem because $\hat{\boldsymbol{w}}_{0, i}, \hat{\boldsymbol{w}}_{1, i}, \boldsymbol{\tau}$, and $\boldsymbol{P}_{i}$ are coupled with each other. To solve P1, we introduce some new variables and apply the SDR technique [11]. First, we introduce $\bar{R}_{b d, i}$, $\hat{R}_{b d, i}, e_{0, i}$, and let $e_{1, i}=P_{1, i} \tau_{2}, e_{2, i}=P_{2, i} \tau_{3}, W_{i}=$ $\tau_{0} \hat{\boldsymbol{w}}_{0, i} \hat{\boldsymbol{w}}_{0, i}^{H}$. Hence, we have the following new constraints: C6: $\bar{R}_{b d, i} \leq \tau_{1} \log _{2}\left(1+\xi P\left|\alpha_{0, i}\right|^{2}\left|h_{1,3}\right|^{2}\left|\boldsymbol{h}_{0,1}^{H} \hat{\boldsymbol{w}}_{1, i}\right|^{2} / \sigma_{a p}^{2}\right)+$ $\tau_{2} \log _{2}\left(1+\xi e_{1, i}\left|h_{2,3}\right|^{2} /\left(\sigma_{a p}^{2} \tau_{2}\right)\right), \mathrm{C} 7: \bar{R}_{b d, i} \leq \tau_{1} \log _{2}(1+$ $\left.\xi P\left|\alpha_{0, i}\right|^{2}\left|h_{1,2}\right|^{2}\left|\boldsymbol{h}_{0,1}^{H} \hat{\boldsymbol{w}}_{1, i}\right|^{2} / \sigma_{h d}^{2}\right)$, C8: $\hat{R}_{b d, i}=\tau_{0} \log _{2}(1+$ $\left.\xi P\left|\alpha_{0, i}\right|^{2}\left|h_{1,3}\right|^{2} e_{0, i} /\left(\sigma_{a p}^{2} \tau_{0}\right)\right), \quad$ C9: $\quad R_{h d, i}=\tau_{3} \log _{2}(1+$ $\left.\xi e_{2, i}\left|h_{2,3}\right|^{2} /\left(\sigma_{a p}^{2} \tau_{3}\right)\right), \mathrm{C} 10: e_{0, i} \leq \operatorname{Tr}\left(\boldsymbol{h}_{0,1} \boldsymbol{h}_{0,1}^{H} \boldsymbol{W}_{i}\right), \mathrm{C} 11: e_{1, i}+$ $e_{2, i} \leq \eta P \operatorname{Tr}\left(\boldsymbol{h}_{0,2} \boldsymbol{h}_{0,2}^{H} \boldsymbol{W}_{i}\right), \mathrm{C} 12: \operatorname{Tr}\left(\boldsymbol{W}_{i}\right) \leq \tau_{0}, \mathrm{C} 13: \boldsymbol{W}_{i} \geq 0$, and C14: $\operatorname{rank}\left(\boldsymbol{W}_{i}\right)=1$. Then, $\mathbf{P 1}$ is recast as

$$
\begin{aligned}
\max _{\boldsymbol{W}_{i}, \hat{\boldsymbol{w}}_{1, i}, \boldsymbol{e}_{i}, \boldsymbol{\tau}, \hat{R}_{b d, i}, \bar{R}_{b d, i}, R_{h d, i}} \omega_{1}\left(\hat{R}_{b d, i}+\bar{R}_{b d, i}\right)+\omega_{2} R_{h d, i} \\
\text { s.t. C1, C2, C5, C6, C7, C8, C9,C10, C11, C12, C13, C14, }
\end{aligned}
$$

where $\boldsymbol{e}_{i}=\left[e_{0, i}, e_{1, i}, e_{2, i}\right]$. However, Problem $\mathbf{P 2}$ is still not a convex optimization problem due to the rank-one constraint and the couple of $\tau_{1}$ and $\hat{\boldsymbol{w}}_{1, i}$. To handle this, we first give the following proposition. Denote the optimal solution for $\mathbf{P 2}$ as $\left\{\boldsymbol{W}_{i}^{*}, \hat{\boldsymbol{w}}_{1, i}^{*}, \boldsymbol{e}_{i}^{*}, \boldsymbol{\tau}^{*}, \hat{R}_{b d, i}^{*}, \bar{R}_{b d, i}^{*}, R_{h d, i}^{*}\right\}$, where $e_{i}^{*}=$ $\left[e_{0, i}^{*}, e_{1, i}^{*}, e_{2, i}^{*}\right]$ and $\tau^{*}=\left[\tau_{0}^{*}, \tau_{1}^{*}, \tau_{2}^{*}, \tau_{3}^{*}\right]$.

Proposition 1. The optimal energy beamforming vector during $\tau_{1}$ is given by $\hat{\boldsymbol{w}}_{1, i}^{*}=\boldsymbol{h}_{0,1} /\left\|\boldsymbol{h}_{0,1}\right\|$.

The proof of Proposition 1 can be done by contradiction theory and is omitted due to the limited space. Then, based on Proposition 1, C6 and C7 are recast as C15: $\bar{R}_{b d, i} \leq \tau_{1} \log _{2}(1+$ $\left.\xi P\left|\alpha_{0, i}\right|^{2}\left|h_{1,3}\right|^{2}|| \boldsymbol{h}_{0,1}||^{2} / \sigma_{a p}^{2}\right)+\tau_{2} \log _{2}\left(1+\xi e_{1, i}\left|h_{2,3}\right|^{2} /\left(\sigma_{a p}^{2} \tau_{2}\right)\right)$ and C16: $\bar{R}_{b d, i} \leq \tau_{1} \log _{2}\left(1+\xi P\left|\alpha_{0, i}\right|^{2}\left|h_{1,2}\right|^{2}|| \boldsymbol{h}_{0,1}||^{2} / \sigma_{h d}^{2}\right)$, respectively. With Proposition $1, \mathbf{P 2}$ is still non-convex due to the rank-one constraint. The SDR technique is an efficient approximation technique to convert the non-convex problem to a convex problem [11]. By relaxing C14 following SDR, $\mathbf{P 2}$ is recast as follows:

$$
\begin{gathered}
\max _{W_{i}, \boldsymbol{e}_{i}, \boldsymbol{\tau}, \hat{R}_{b d, i}, \bar{R}_{b d, i}, R_{h d, i}} \omega_{1}\left(\hat{R}_{b d, i}+\bar{R}_{b d, i}\right)+\omega_{2} R_{h d, i} \\
\text { s.t. } \quad \mathrm{C} 1, \mathrm{C} 2, \mathrm{C} 8, \mathrm{C} 9, \mathrm{C} 10, \mathrm{C} 11, \mathrm{C} 12, \mathrm{C} 13, \mathrm{C} 15, \mathrm{C} 16 .
\end{gathered}
$$

Proposition 2. Problem P3 is a convex problem [12].

According to Proposition 2, the optimal solution for P3 can be solved by some standard optimization techniques. In this paper, we use CVX tools [13] to derive the optimal solution. The optimal power allocations are further given by
$P_{1, i}^{*}=e_{1, i}^{*} / \tau_{2}^{*}$ and $P_{2, i}^{*}=e_{2, i}^{*} / \tau_{3}^{*}$. Then, we compute the optimal solution $\hat{\boldsymbol{w}}_{0, i}^{*}$ from $\boldsymbol{W}_{i}^{*}$. Note that if $\boldsymbol{W}_{i}^{*}$ satisfies the rank-one constraint, $\hat{\boldsymbol{w}}_{0}^{*}$ computed from $\boldsymbol{W}_{i}^{*} / \tau_{0}^{*}$ by eigendecomposition is the optimal energy beamforming vector during $\tau_{0}$. Hence, we proceed to show that $\boldsymbol{W}_{i}^{*}$ always has the rank-one property in the following proposition.

Proposition 3. The optimal solution $\boldsymbol{W}_{i}^{*}$ derived from $\boldsymbol{P 3}$ is a rank-one matrix.

Proof. To show $\boldsymbol{W}_{i}^{*}$ is a rank-one matrix, we first give the following optimization problem.

$$
\begin{array}{ll}
\min _{\boldsymbol{W}_{i}} & \operatorname{Tr}\left(\boldsymbol{W}_{i}\right) \\
\text { s.t. } & e_{0, i}^{*} \leq \operatorname{Tr}\left(\boldsymbol{h}_{0,1} \boldsymbol{h}_{0,1}^{H} \boldsymbol{W}_{i}\right), \quad \boldsymbol{W}_{i} \geq 0, \\
& e_{1, i}^{*}+e_{2, i}^{*} \leq \eta P \operatorname{Tr}\left(\boldsymbol{h}_{0,2} \boldsymbol{h}_{0,2}^{H} \boldsymbol{W}_{i}\right) .
\end{array}
$$

Denote the optimal solution for Problem $\mathbf{P 4}$ as $\boldsymbol{W}_{i}^{\dagger}$, which is also a feasible solution for $\mathbf{P 3}$. The reason is that there are more constraints in $\mathbf{P 3}$ than in $\mathbf{P 4}$, which guarantees that a feasible solution for $\mathbf{P 3}$ is also feasible for $\mathbf{P 4}$. Hence, we can derive that $\operatorname{Tr}\left(\boldsymbol{W}_{i}^{\dagger}\right) \leq \operatorname{Tr}\left(\boldsymbol{W}_{i}^{*}\right) \leq \tau_{0}^{*}$, which shows that $\boldsymbol{W}_{i}^{\dagger}$ is a feasible solution for P3. Furthermore, since the objective function of $\mathbf{P 3}$ is a function of $e_{i}, \tau, \hat{R}_{b d, i}, \bar{R}_{b d, i}$ and $R_{h d, i}$, we can derive that $\left\{\boldsymbol{W}_{i}^{\dagger}, \boldsymbol{e}_{i}^{*}, \tau^{*}, \hat{R}_{b d, i}^{*}, \bar{R}_{b d, i}^{*}, R_{h d, i}^{*}\right\}$ is also the optimal solution for P3, i.e., $\boldsymbol{W}_{i}^{\dagger}=\boldsymbol{W}_{i}^{*}$. According to the theorem given in [[14], Theorem 3.2], we then show that $\boldsymbol{W}_{i}^{\dagger}$ is a rank-one matrix. Since there exists an optimal solution $\boldsymbol{W}_{i}^{\dagger}$ satisfying $\left(\operatorname{rank}\left(\boldsymbol{W}_{i}^{\dagger}\right)\right)^{2} \leq 2$, we derive that $\boldsymbol{W}_{i}^{\dagger} \neq 0$ is rank-one. Hence, $\operatorname{rank}\left(\boldsymbol{W}_{i}^{*}\right)=1$.

\section{B. Case ii}

Similar to the first case, we add the following constraints: C17: $\sum_{i=0}^{2} t_{i} \leq 1, \mathrm{C} 18: t_{i} \geq 0, \forall i, \mathrm{C} 19: P_{1, i i} t_{1} \leq E_{h, i i}$, C20: $\left|\hat{\boldsymbol{w}}_{0, i i}\right|^{2} \leq 1$, and C21: $\left|\hat{\boldsymbol{w}}_{2, i i}\right|^{2} \leq 1$. Then, the optimization problem for Case $i i$ is formulated by:

$$
\begin{array}{cl}
\max _{\hat{\boldsymbol{w}}_{i i}, P_{1, i i}, t} & \omega_{1} R_{b d, i i}+\omega_{2} R_{h d, i i} \\
\text { s.t. } & \mathrm{C} 17, \mathrm{C} 18, \mathrm{C} 19, \mathrm{C} 20, \mathrm{C} 21,
\end{array}
$$

where $\hat{\boldsymbol{w}}_{i i}=\left[\hat{\boldsymbol{w}}_{0, i i}, \hat{\boldsymbol{w}}_{2, i i}\right]$ and $\boldsymbol{t}^{*}=\left[t_{0}^{*}, t_{1}^{*}, t_{2}^{*}\right]$ Following the similar approach for Case $i$, Problem P5 can be solved as follows. We introduce auxiliary variables $\bar{R}_{h d, i i}, e_{0, i i}, e_{1, i i}$, and let $e_{2, i i}=P_{1, i i} t_{1}$, $\boldsymbol{W}_{i i}=t_{0} \hat{\boldsymbol{w}}_{0, i i} \hat{\boldsymbol{w}}_{0, i i}^{H}$. Then, we introduce the following new constraints C22: $t_{1} \log _{2}\left(1+\xi e_{2, i i}\left|h_{2,3}\right|^{2} /\left(\sigma_{a p}^{2} t_{1}\right)\right)+$ $t_{2} \log _{2}\left(1+\xi P\left|\alpha_{2, i i}\right|^{2}\left|h_{1,3}\right|^{2}\left|\boldsymbol{h}_{0,1}^{H} \hat{\boldsymbol{w}}_{2, i i}\right|^{2} / \sigma_{a p}^{2}\right) \geq \bar{R}_{h d, i i}$, C23: $\quad t_{1} \log _{2}\left(1+\xi e_{2, i i}\left|g_{1,2}\right|^{2} /\left(\sigma_{b d}^{2} t_{1}\right)\right) \geq \bar{R}_{h d, i i}$, $\mathrm{C} 24: e_{0, i i} \leq \operatorname{Tr}\left(\boldsymbol{h}_{0,2} \boldsymbol{h}_{0,2}^{H} \boldsymbol{W}_{i i}\right), \mathrm{C} 25: e_{1, i i} \leq \operatorname{Tr}\left(\boldsymbol{h}_{0,1} \boldsymbol{h}_{0,1}^{H} \boldsymbol{W}_{i i}\right)$, C26: $R_{b d, i i}=t_{0} \log _{2}\left(1+\xi P\left|\alpha_{0, i i}\right|^{2}\left|h_{1,3}\right|^{2} e_{1, i i} /\left(\sigma_{a p}^{2} t_{0}\right)\right)$, C27: $e_{2, i i} \leq \eta P e_{0, i i}, \mathrm{C} 28: \operatorname{Tr}\left(\boldsymbol{W}_{i i}\right) \leq \tau_{0}, \mathrm{C} 29: \boldsymbol{W}_{i i} \geq 0$, and C30: $\operatorname{rank}\left(\boldsymbol{W}_{i i}\right)=1$. Then, $\mathbf{P 5}$ is recast as:

$$
\begin{array}{r}
\max _{\boldsymbol{i} i,}, \hat{\boldsymbol{w}}_{2, i i}, \boldsymbol{e}_{i i}, \boldsymbol{t}, \boldsymbol{R}_{b d, i i}, \bar{R}_{h d, i i} \\
\text { s.t. C17, C18,C21, C22, C23, C24, } \\
\text { C25, C26,C27, C28, C29, C30, }
\end{array}
$$


where $e_{i i}=\left[e_{0, i i}, e_{1, i i}, e_{2, i i}\right]$. Denote the optimal solution for Problem P6 as $\left\{\boldsymbol{W}_{i i}^{*}, \hat{\boldsymbol{w}}_{2, i i}^{*}, \boldsymbol{e}_{i i}^{*}, \boldsymbol{t}^{*}, R_{b d, i i}^{*}, \bar{R}_{h d, i i}^{*}\right\}$, where $e_{i i}^{*}=$ $\left[e_{0, i i}^{*}, e_{1, i i}^{*}, e_{2, i i}^{*}\right]$, and $\boldsymbol{t}^{*}=\left[t_{0}^{*}, t_{1}^{*}, t_{2}^{*}\right]$. Similar to Case $i$, we can derive the following Proposition 4.

Proposition 4. The optimal energy beamforming design during $t_{2}$ is given by $\hat{\boldsymbol{w}}_{2, i i}^{*}=\boldsymbol{h}_{0,1} /\left\|\boldsymbol{h}_{0,1}\right\|$.

Based on Proposition 4, $\mathrm{C} 22$ is rewritten as C31: $t_{1} \log _{2}\left(1+\xi e_{2, i i}\left|h_{2,3}\right|^{2} /\left(\sigma_{a p}^{2} t_{1}\right)\right)+t_{2} \log _{2}(1+$ $\left.\xi P\left|\alpha_{2, i i}\right|^{2}\left|h_{1,3}\right|^{2}\left|\boldsymbol{h}_{0,1}\right|^{2} / \sigma_{a p}^{2}\right) \geq \bar{R}_{h d, i i}$. P6 is then reformulated as P7 without considering C30.

$$
\max _{\boldsymbol{W}_{i i}, \boldsymbol{e}_{i i}, \boldsymbol{t}, R_{b d, i i}, \bar{R}_{h d, i i}} \omega_{1} R_{b d, i i}+\omega_{2} \bar{R}_{h d, i i}
$$

$$
\text { s.t. C17, C18, C23, C24, C25,C26, C27, C28, C29, C31. }
$$

It can be proved that $\mathbf{P 6}$ is a convex problem [12], hence the optimal solution of which be solved by CVX tools [13]. Based on the derived solution, the optimal power allocation is given by $P_{1, i i}^{*}=e_{2, i i}^{*} / t_{1}^{*}$, and the optimal energy beamforming vector during $t_{0}$ is derived from $\boldsymbol{W}_{i i}^{*} / t_{0}^{*}$ by eigen-decomposition since $\boldsymbol{W}_{i i}^{*}$ is a rank-one matrix.

\section{Simulation Results}

In this section, simulation results are given to evaluate the performance of the proposed schemes. The simulated network topology is a 2-D plane, where the position of each node is described with its coordinate $(x, y)$. The coordinates of the $\mathrm{PB}$, the AP and the two devices are given as $(0,10),(10,0),(0,0)$, and $(2,1)$, respectively. All channels are modeled following Rayleigh fading with distribution $C \mathcal{N}\left(0, d_{m, n}^{-\kappa}\right)$, where $\kappa$ denotes the path-loss exponent and is set at 2 , and $d_{m, n}$ is the distance between two nodes $m, n$. We assume $\sigma_{a p}^{2}=\sigma_{h d}^{2}=$ $\sigma_{b d}^{2}=-40 \mathrm{dBm}, \eta=0.7,\left|\alpha_{0, i}\right|^{2}=\left|\alpha_{0, i i}\right|^{2}=\left|\alpha_{2, i i}\right|^{2}=1$, $\xi=-5 \mathrm{~dB}$ [7], and $N=10$. The proposed schemes under Case $i$ and Case $i i$ are denoted as 'proposed scheme $i$ ' and 'proposed scheme $i i$ ', respectively. The scheme that both devices are the HD devices [3] and the non-cooperation schemes for Case $i$ and Case $i i$ are used as the benchmark schemes.

Fig. 2a shows the WSR versus $P$ with $\omega_{1}=\omega_{2}=0.5$. It can be observed that the results obtained by two proposed schemes are superior to those of the benchmark schemes. This is because the time of information delivery is extended since the dedicated $\mathrm{EH}$ phases for both proposed schemes are not required and user cooperation can enhance the system WSR. Moreover, the WSR of the proposed scheme $i i$ is larger than that of $i$. This is because for the proposed scheme $i$, the channel conditions of the BD for IB are worse and the harvested energy of the $\mathrm{HD}$ are used for transmitting its own information and forwarding the information of the BD, which limits the WSR. While, for the proposed scheme $i i$, the channel conditions of the $\mathrm{BD}$ for IB are better, and the harvested energy of the HD is only used for its own IT. Hence, the proposed scheme $i i$ can achieve a larger WSR. Fig. 2b shows the effect of $\omega_{1}$ on the system WSR with $P=20 \mathrm{dBm}$. From Fig. $2 \mathrm{~b}$, we observe that the WSR of the proposed schemes are larger than those of the benchmark schemes, which shows the superiority of the proposed schemes. Since changing $\omega_{1}$ can guarantee

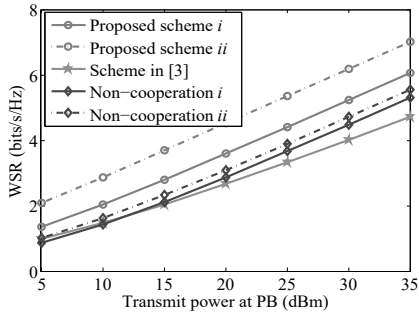

(a) WSR versus $P$.

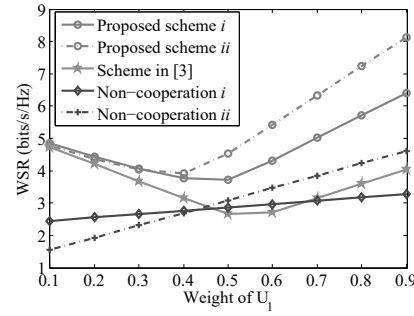

(b) WSR versus $\omega_{1}$.
Fig. 2: Performance evaluation.

user fairness, we conclude that guaranteeing user fairness may degrade the system WSR.

\section{CONCLUSION}

We have proposed two user cooperation schemes in the WPCN with BackCom, where one device is the BD and another device is the HD. We have considered two cases in which either $\mathrm{HD}$ or $\mathrm{BD}$ is located nearer the $\mathrm{AP}$ and it can serve as the relay node for another node in forwarding information to the AP. Two WSR optimization problems have been formulated to jointly optimize the time schedule, power allocation, and energy beamforming vectors. Then, the variable substitutions and SDR technique have been developed to obtain the optimal solution. Finally, simulation results have been provided to evaluate the efficiency of the proposed schemes.

\section{REFERENCES}

[1] H. Ju et al., "Throughput maximization in wireless powered communication networks," IEEE TWC, vol. 13, no. 1, pp. 418-428, Jan. 2014.

[2] $\mathrm{H}$. Ju et al., "User cooperation in wireless powered communication networks," in IEEE GLOBECOM, USA, Dec. 2014, pp. 1430-1435.

[3] X. Di et al., "Optimal resource allocation in wireless powered communication networks with user cooperation," IEEE TWC, vol. 16, no. 12, pp. 7936-7949, Dec. 2017

[4] G. Zhu et al., "Inference from randomized transmissions by many backscatter sensors," IEEE TWC, vol. 17, no. 5, pp. 3111-3127, May 2018.

[5] S. Gong, et al., "Backscatter relay communications powered by wireless energy beamforming," IEEE TCOM, vol. 66, no. 7, pp. 3187-3200, July 2018.

[6] D. T. Hoang et al., "Ambient backscatter: A new approach to improve network performance for RF-powered cognitive radio networks," IEEE TCOM, vol. 65, no. 9, pp. 3659-3674, Sept. 2017.

[7] S. H. Kim et al., "Hybrid backscatter communication for wirelesspowered heterogeneous networks," IEEE TWC, vol. 16, no. 10, pp. 65576570, Oct. 2017.

[8] D. Bharadia et al., "BackFi: High throughput WiFi backscatter," in SIGCOMM, London, UK, Aug. 2015, pp. 283-296.

[9] G. Wang et al., "Ambient backscatter communication systems: Detection and performance analysis," IEEE TCOM, vol. 64, no. 11, pp. 4836-4846, Nov. 2016.

[10] Y. Liang et al., "Gaussian orthogonal relay channels: Optimal resource allocation and capacity," IEEE Trans. Inform. Theory, vol. 51, no. 9, pp. 3284-3289, Sept. 2005

[11] Z. Q. Luo et al., "Semidefinite relaxation of quadratic optimization problems," IEEE Signal Process., vol. 27, no. 3, pp. 20-34, May 2010.

[12] S. Boyd et al., Convex Optimization. Cambridge University Press, 2004.

[13] M. Grant et al., "CVX: MATLAB software for disciplined convex programming," [Online], Available: http://cvxr.com/cvx

[14] Y. Huang et al., "Rank-constrained separable semidefinite programming with applications to optimal beamforming, "IEEE Trans. Signal Process., vol. 58, no. 2, pp. 664-678, Feb. 2010. 